\title{
A SIMPLE PLATE/SHELL TRIANGLE
}

\author{
G.PRATHAP \\ Structural Sciences Division, National A eronautical Laboratory, Bangalore, India
}

SUMMARY

A simple shear flexible triangular plate/shell element with three/five displacement degrees-of-freedom at the three corner nodes only, is identified. It does not lock or have zero-energy mechanisms. The specific geometrical configurations in which this is possible are derived through field consistency patch tests.

\section{INTRODUCTION}

The search for and the apparent elusiveness of a simple $\mathrm{C}^{\circ}$ shear flexible triangle for plate/shell structures is well documented.' It is clear from these exercises that it may not be possible to have a general triangle that does not lock or even identify a single integration point or reduced integration rule that can remove locking. In this paper, it is shown that a right-angled triangle, correctly aligned and with correctly chosen integration points, will not lock or have zero-energy mechanisms.

\section{DESIGN OF ELEMENT}

\section{Introductory remarks}

The shear flexible plate/shell problem is a multi-field continuum problem. The plate is described by the variables $\mathrm{w}, 0$ and $/ 3$ and the shell by $\mathrm{u}, \mathrm{v}, \mathrm{w}, 0$ and $/ 3$, the displacements and rotations at the middle. surface. In certain limiting situations, some of its strain fields are constrained, e.g. shear strains -+ 0 in the Kirchhoff sense and membrane strains -*0 in the inextensional shell case. These strain fields comprise terms of different derivative orders from the contributing field variables. Thus $\mathrm{y}_{\mathrm{x}_{-}}=0-\mathrm{w}, \mathrm{x}, a_{x}=u,+w / R_{\mathrm{x}}$, etc: A recent exercise ${ }^{10}$ showed that membrane and shear locking arose because these constrained strain fields are not described 'consistently' if equal order interpolations for all field variables $u$ to /3 are used. Typically, in a rectangular shell element, with field interpolations of the type

$$
\begin{aligned}
& \boldsymbol{u}=a_{o}+a_{l} x+a_{2} y+a_{3} x y \\
& w=c_{0}+c_{1} \mathbf{x}+c_{2} y+c_{3} x y \\
& O=d_{o}+d_{l} x+d_{2} y+d_{3} x y
\end{aligned}
$$

we have strain fields such as

$$
\begin{aligned}
y x \sim & =(d o-c 1)+(d 2-c 3) Y+d 1 x+d 3 x y \\
\mathrm{Ex} & =(a 1+c o / R x)+(a 3+c 2 / R x) Y+(c l / R x) x+(c 3 / R x) x y
\end{aligned}
$$

It is seen that, in these strain fields, two of the coefficients comprise contributions from both field 
variables ('consistent' representation) and two have terms from only one field variable ('inconsistent' representation). It was shown recently' that an exact integration of the energy terms from these strain fields will produce 'true constraints' from the 'consistent' terms and 'spurious constraints' from the 'inconsistent' terms. The latter cause shear and membrane locking.

It was also found that techniques such as reduced integration, reduced interpolation, addition of incompatible modes, etc., that can produce a strain energy from the constrained fields in terms of consistent quantities alone, usually produced elements that would not lock. We shall see that field consistency for strain fields as shown earlier does not suffice for the linear triangle, which seems to have additional problems of its own, associated with the linear interpolation within a triangular field.

\section{The linear triangle}

Consider the triangular element oriented arbitrarily as shown in Fig. 1. Typical interpolations are of the form

$$
\begin{aligned}
& w=c_{\circ}+c_{1} x+c_{2} y \\
& \sigma=d_{\circ}+d_{1} x+d_{2} y
\end{aligned}
$$

and a typical constrained strain field becomes

$$
y x .=(d o-c l)+d 1 x+d 2 Y
$$

An exact integration of the shear strain energy will produce spurious constraints arising from the $d$, and $d_{2}$ terms. This explains the dramatically poor behaviour of exactly integrated $\mathrm{C}^{\circ}$ triangles.

The arguments related to field consistency ${ }^{10}$ recommend a reduced integration of the energies from the constrained strain fields. This can be readily performed with a 1-point integration. This, it is expected, will retain only consistently represented strains such as $\mathrm{y}_{\mathrm{SZ}}=\left(d_{\circ}-c_{1}\right)$ and should therefore not lock. An experiment using the centroid as integrating point (the SRI triangle) ended, surprisingly, in failure.' Subsequently, it was shown that SRI triangles can generate locking even if, within the element, the strain description is consistent.' Many experiments to locate an optimal integration point have failed."

Clearly, locking in a linear triangle originates because it is unable to respond to certain basic or minimum strain fields which are essential for a Kirchhoff-type bending action. By applying the field consistency criteria to patch tests of a general triangle, we can derive the form of an element that would not lock.

\section{Application of field tests}

We restrict attention to the design of field consistent shear strains under Kirchhoff bending fields. The arguments for inextensional shell action are identical and produce identical requirements.

In the thin plate/shell limit, the general triangle shown in Figure 1 must respond to each of the three fields described by $w=A x^{e}, w=B x y$ and $\mathrm{w}=\mathrm{Cy}^{2}$. We now apply in turn, the nodal values $\mathrm{w}_{\mathrm{i}}$, $0_{\mathrm{i}}$ and $13_{\mathrm{i}}$ arising from these fields to the triangle and compute the strain fields at an integrating point $(\mathrm{x}, \mathrm{y}$,$) . Simple algebraic manipulations give$

$$
\begin{array}{ll}
\text { (AX) } & y x Z(A)=B-w,{ }_{x}=A\left[2 x,-E b_{i} x ?\right] \\
(\mathrm{BX}) & y x z(B)=B-\mathrm{w},{ }_{x}=B\left[y,-E b_{i x i y i}\right] \\
(C X) & y, 2(C)=O-w,=C E b_{i} y ?
\end{array}
$$




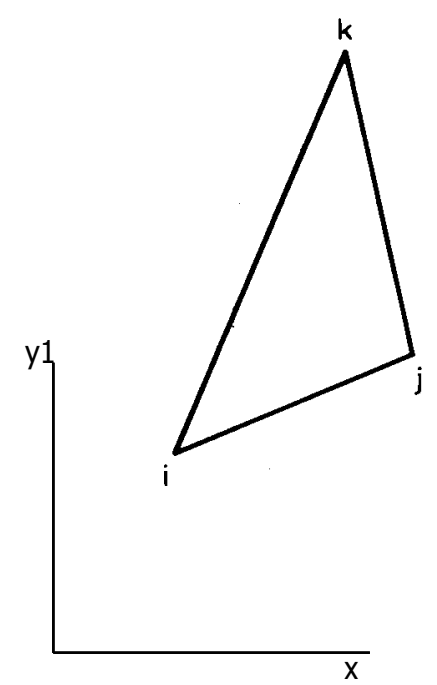

Figure 1 . The arbitrary linear triangle

(CY) $\quad y_{\mathrm{yz}}(\mathrm{C})=/ 3-w,=C\left[^{2} Y r^{\text {Eciy; }]}\right.$

\$BY) $\quad y_{y} .(B)=/ 3-w, y=B\left[x_{1}-\mathrm{Ec}_{\mathrm{i}} \mathrm{X}_{\mathrm{i}} \mathrm{y}_{\mathrm{i}}\right]$

(AY) $\quad \mathrm{y}_{\mathrm{yz}}(\mathrm{A}) \quad \mathrm{w},{ }_{\mathrm{y}}=\mathrm{AEc}_{\mathrm{i}} \mathrm{x}$ ?

$\mathrm{b}_{\mathrm{i}}=\left(\mathrm{y} ;-\mathrm{y}_{k}\right) / 2 \mathrm{~A}, \mathrm{c}_{\mathrm{i}}=\left(\mathrm{x}_{k}-\mathrm{x},\right) / 2 \mathrm{~A}$, etc., $2 \mathrm{~A}=\mathrm{Ex}_{\mathrm{i}}\left(\mathrm{y}_{j}-\mathrm{y}_{k}\right)$, where the summation is taken over 3 , in a cyclic order. It is necessary that, at the integrating point, all these strains should vanish so that no spurious strain energies emerge from these field tests.

From equations (AY) and (CX) we have the conditions

$$
\begin{aligned}
& \mathrm{Ec}_{\mathrm{i}} \mathrm{x} ?=0 \\
& \mathrm{~Eb}_{\mathrm{i}} \mathrm{y} ?=0
\end{aligned}
$$

prhich, after a little manipulation, leads to

$$
\begin{array}{r}
(\mathrm{xi}-\mathrm{xi})(\mathrm{x} ;-x,)\left(x_{k}-\mathrm{xi}\right)=0 \\
(Y i-Y ;)(Y ;-Y k)(Y k-\mathrm{Yi})=0
\end{array}
$$

leading to the inevitable conclusion that only a triangle which has at least one edge aligned with the $\mathrm{x}$-axis and at least one edge aligned with the $\mathrm{y}$-axis will admit zero shear strains from these fields at any integrating point. This is a condition independent of the choice of integrating point.

We next examine the conditions (BX) and (CY) and (AX) and (BY) to see if any conflict arises if a single integrating point is used for the $\mathrm{x}$-axis and $\mathrm{y}$-axis related terms. Again, after some algebraic pianipulations, the conditions reduce to

$$
\begin{aligned}
& \operatorname{Exi}\left[(\mathrm{Y} ;-y i)^{2}+(Y k-y i)^{2}\right]=0 \\
& \operatorname{EYi}\left[\left(\mathrm{x} ;-\mathrm{x}^{i}\right)^{2}+\left(\mathrm{x}_{k}-\mathrm{x}_{\mathrm{i}}\right)^{2}\right]=0
\end{aligned}
$$

For any general triangle, these conditions are satisfied only if $\mathrm{x}_{\mathrm{i}}=\mathrm{x}_{\mathbf{j}}=\mathrm{x}_{k}$ and $\mathrm{y}_{\mathrm{i}}=\mathrm{y}_{\mathbf{j}}=\mathrm{y}_{k}$, i.e. if the triangle shrinks to a point. A single integrating point cannot be found for a finite triangle.

It is obvious therefore that two integrating points $\left(\mathrm{x} ; y_{x}\right)$ and $\left(\mathrm{x}_{\mathrm{y}}, \mathrm{y},\right)$ must now be found from the 
conditions $(\mathrm{AX})$ to $(\mathrm{CX})$ and $(\mathrm{AY})$ to $(\mathrm{CY})$, respectively. They are therefore

$$
\begin{aligned}
x_{x} & =1 / 2 E b ; x ? \\
Y x & =E b 1 x j y j \\
b ; b ; b_{k} & =0 \\
x_{y} & =E c ; x ; y, \\
Y_{31} & =1 / 2 E c ; x ? \\
C, C^{\prime} c_{k} & =0
\end{aligned}
$$

Since we have already established that the triangle must have at least one of the $b, s^{\prime}=0$ and at least one of the $c_{i}^{\prime} s=0$, we get for say $b ;=0$ and $c_{j}=0$,

$$
\begin{aligned}
& \mathrm{x}_{\mathrm{x}}=1 / 2\left(x,+x_{3}\right) \\
& \mathrm{Yx}=\mathrm{Yi}=\mathrm{Y} ; \\
& \mathrm{x}_{\mathrm{y}}=\mathrm{x} ; \quad \mathrm{xk} \\
& y_{\mathrm{y}}={ }^{1} / 2(\mathrm{Y} ;+\mathrm{Yk})
\end{aligned}
$$

Thus, the optimal triangular element is a right-angled triangle, with its sides containing the rightangle aligned with the $\mathrm{x}$ and $\mathrm{y}$ axes, and having the mid-points of these sides as the integration points for the respective constrained strain fields.

Numerical experiments in the next section confirm that such elements have no membrane or shear locking effects or zero-energy mechanisms.

\section{NUMERICAL EXPERIMENTS}

Four numerical test cases are used to demonstrate the acceptability of the proposed plate/shell element. Several element variations are used to show clearly how the effects of shear and membrane locking operate. The element variations are:

1. OMOS-the optimal triangle proposed here, with membrane and shear strains evaluated at the optimal points.

2. SRI-the selectively reduced integrated triangle of Reference 5, which uses a single integrating point at the centroid for both membrane and shear strains.

3. EMES-the exactly integrated triangle, using exact integration of membrane and shear energy. 4. OMES-a triangle with the membrane strains evaluated only at the optimal point, whereas the shear energy is exactly integrated.

5. EMOS-a triangle with the membrane energy exactly integrated, whereas the shear strains are evaluated at the optimal point.

\section{Simply-supported square plate with uniform distributed load}

A square plate, simply supported on all edges, of sides $\mathrm{L}=10$ in., $\mathrm{E}=10.92 \times 10^{5}$ psi., $v=0^{3}$ under uniformly distributed load is considered. Symmetry allows a quarter plate to be modelled by a uniform $4 \times 4$ grid of rectangles, each comprising two triangles with the diagonals running in a north-east direction. A measure of shear locking is obtained using the additional stiffening parameter $^{15} e$, where

$$
\boldsymbol{e}-\frac{\mathrm{w}(\text { theory })}{\mathrm{w}(\mathrm{fem})}-1
$$




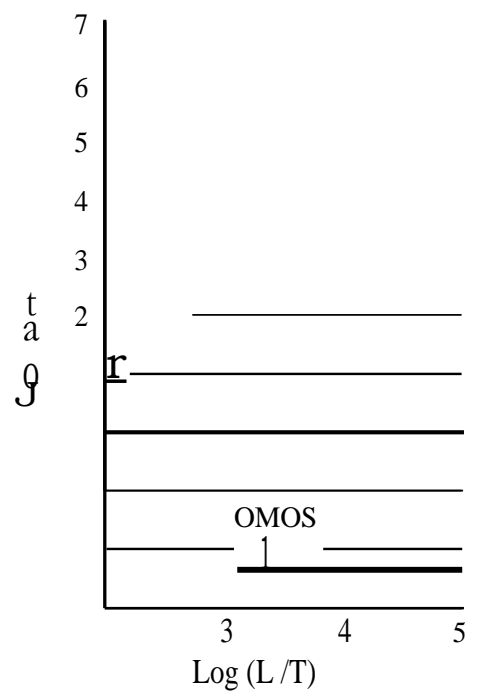

Figure 2. Additional stiffening parameter for a simply-supported square plate under uniform distributed load

In this case, $\mathrm{w}$ is the central deflection of the plate. If shear locking exists, the error $e$ will propagate in $a(L I T)^{\prime}$ fashion as the thickness $T$ is diminished. Figure 2 shows that this is true for both the exactly integrated and SRI triangles, whereas the present triangle produces very accurate results without any signs of locking.

\section{Twisting of a corner-supported square plate}

This offers a test case for the presence of zero-energy mechanisms which result from an underintegration of the strain energy. The square plate described above is now supported on three cowers and a load of $\mathrm{P}=\mathrm{I} \underline{\mathrm{lbf}}$. is applied at the fourth corner. The entire grid is now idealized in the same manner for the earlier example. The EMES and SRI triangles fail to produce the correct deformation patterns altogether, whereas the present triangle, OMOS, produces almost the exact answer for a wide range of thicknesses, without any evidence of locking or zero-energy mechanisms (see Table I). In comparison, a 4-node quadrilateral with 1-point integration of the shear energy" fails to produce an answer for this example, due to the presence of zero-energy mechanisms.

\section{The pinched cylinder}

This is an example of an inextensional shell problem in which membrane locking is tangibly present. One octant of the cylinder is considered. The geometrical and elastic properties are: radius $=4.953$ in., length $=10.35$ in., $\mathrm{E}=10.5 \times 10^{6} \underline{\mathrm{lbf}}$. in $. \mathrm{z}, \mathrm{v}=0.3125$ and pinching loads of

Table I. Twisting of corner supported plate

\begin{tabular}{|c|c|c|c|c|}
\hline Thickness & OMOS & SRI & EMES & Theory \\
\hline 0. 1000 & $0-1788 \mathrm{E}+0$ & $0 \cdot 2295 E-1$ & $O 2246 E-2$ & $0 \cdot 1789 E+O$ \\
\hline 00100 & $0 \cdot 1786 E+3$ & $0.2131 E+2$ & $O 2759 E-1$ & $0 \cdot 1789 E+3$ \\
\hline 00010 & $0 \cdot 1786 E+6$ & $0.2130 E+O$ & $0 \cdot 2760 E+O$ & $O 1789 E+6$ \\
\hline 00001 & $0 \cdot 1786 E+9$ & $0 \cdot 2130 E+8$ & $0.2760 E+1$ & $O 1789 E+9$ \\
\hline
\end{tabular}




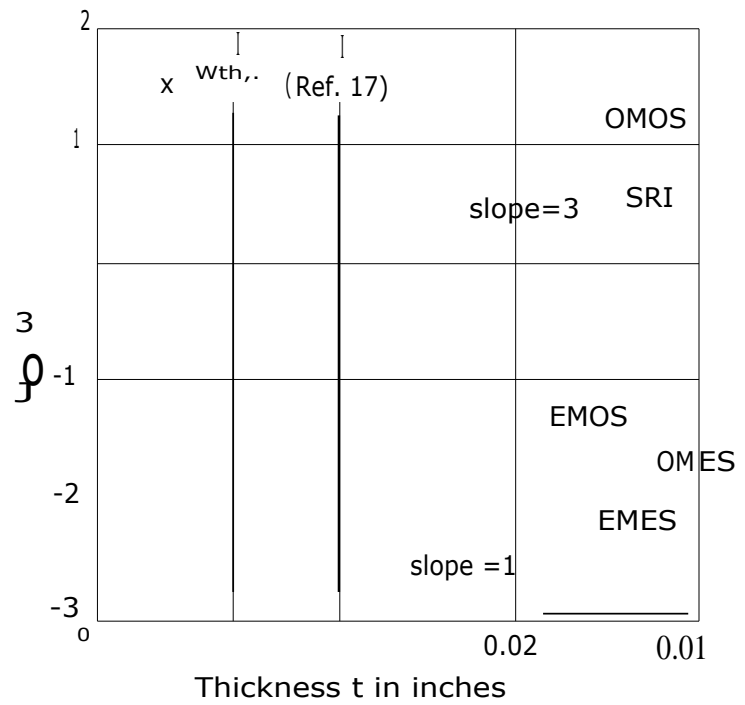

Figure 3. Logarithmic plot of deflection under load vs. thickness for pinched cylinder

$P \quad 1001 b f 0$ are applied. Figure 3 presents the deflection w under the load $P$ obtained from a grid using 16 triangles in a I x 8 mesh. Since a shear flexible deep shell element is used to mode this inextensional thin shell regime, both shear and membrane locking should appear. Figure 3 confirms this. Elements EMOS (in which membrane locking alone in present), OMES (in which shear locking alone is present) and EMES (in which both forms of locking are present) all show an error of the second kind in which the erroneous additional stiffening due to locking varies linearly with the thickness of the shell. Element OMOS gives results which agree well with the theoretical prediction for this problem, $t^{\prime}$ and the deflection and stiffness vary correctly in at' fashion, typical of elements in which no locking is present. The SRI shell element produces results which conform neither to the linear in $t$ variation symptomatic of locking nor to the $t_{3}$ element entirely without locking. A closer examination of the deformation the use of SRI elements shows that a mechanism is also present which combines with locking to give such a result.

\section{The shallow spherical shell}

Figure 4 shows a doubly-curved shallow shell supported on the boundary of a square planform

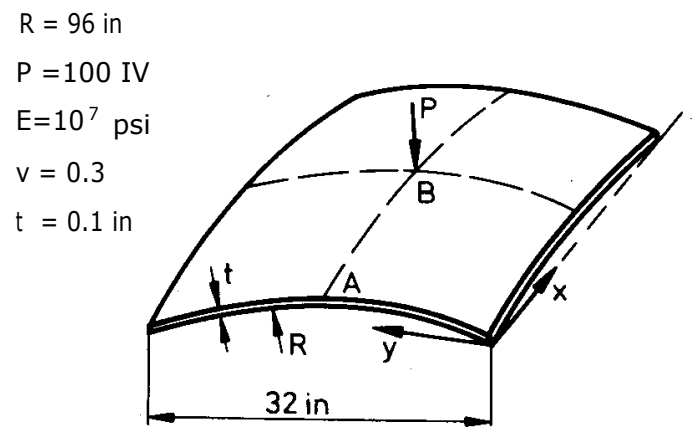

Figure 4. Geometry of spherical cap 

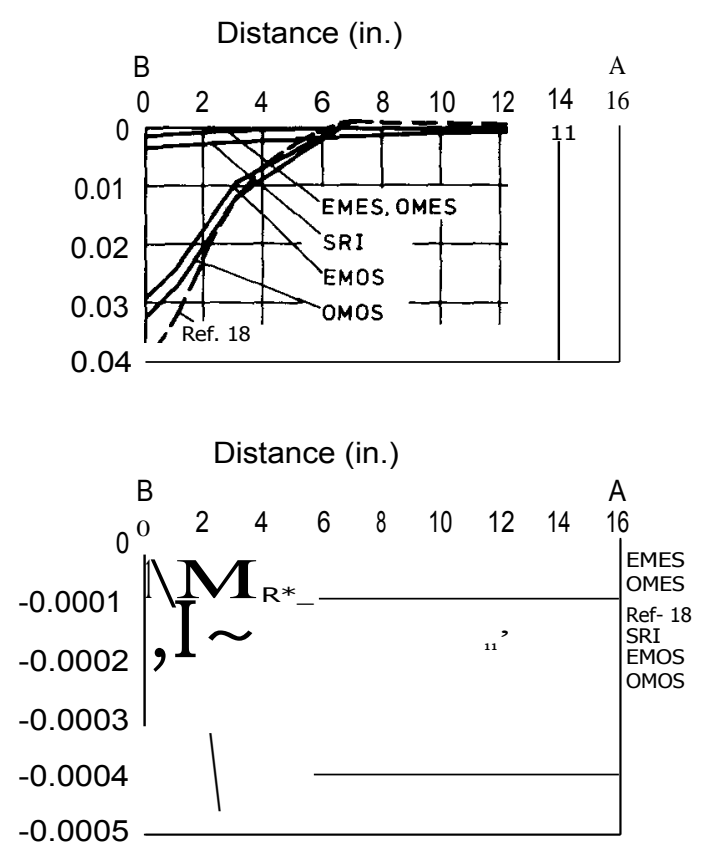

Figure 5. Spherical cap, displacements along AB

with 'diaphragm' conditions on these edges. A quadrant of this shell is modelled by a $4 \times 4$ mesh. Figure 5 shows the normal displacement $\mathrm{w}$ and tangential displacements $\mathrm{u}$ along the line $\mathrm{AB}$ for the various elements considered here. It is clear that the element OMOS does not lock in any way and should converge to the correct answer ${ }^{18}$ as the mesh is refined. Element EMOS shows reasonably good agreement because the effect of membrane locking is less critical in this problem. The other elements show the locking phenomenon and would be unusable in practical situations.

\section{CONCLUSIONS}

The present exercise shows that a field consistent shear flexible linear triangular shell element can be derived only if the triangle is right-angled, is aligned with edges parallel to the rectangular axes and has, as integrating points for the constrained strain fields, the appropriate mid-side points. This exercise also seems to indicate that no general arbitrarily oriented triangle may achieve this. This geometrical restriction does not exclude its use in most practical situations as any domain can be approximated in this fashion-with the hypotenuses of the boundary elements always on the boundary and the sides containing the right-angles always aligned with the $\mathrm{x}$ - and $\mathrm{y}$-axes.

\section{ACKNOWLEDGEMENTS}

The author is very grateful to Mr B. R. Somashekar, Head, Structural Sciences Division, National Aeronautical Laboratory, Bangalore, India and to Dr. Ing. H. W. Bergmann, Director, DFVLR Institute of Structural Mechanics at Braunschweig, W. Germany for their constant help and encouragement. The author is also deeply indebted to DAAD for the award of an Exchange Fellowship which enabled this work to be carried out at Braunschweig. 
1. S. Utku, 'Stiffness matrices for thin triangular elements of non-zero Gaussian curvature', A.I.A.A. J., 5, 1659-1667 (1967).

2. G. A. Wempner, J. T. Oden and D. Kross, `Finite element analysis of thin shells', J. Eng. Mech. Div., ASCE, 94(EM6), 1273-1294(1968).

3. I. Fried, 'Shear in $\mathrm{C}^{\circ}$ and $\mathrm{Cl}$ bending finite elements', Inc. J. Solids Struct., 9, 449-460 (1973).

4. K. Rohwer, 'Development of comparable triangular shell finite elements', DFVLR-Forschungsbericht, 79, 25 (1979).

5. J. L. Batoz, K. J. Bathe and L. W. Ho, 'A study of three-node triangular plate bending elements', Int. j. numer. methods eng., 15, 1771-1812 (1980).

6. T. J. R. Hughes and R. L. Taylor, 'The linear triangular plate bending element', Proc. MAFELAP 1981 Conf., Brunel University, Apr. 28-May 1 (1981).

7. H. Stolarski, T. Belytschko and N. Carpenter, 'Bending and shear-mode decomposition in $\mathrm{C}^{\circ}$ structural elements', J. Struct. Mech., 11, 153-176 (1983).

S. S. Viswanath and G. Prathap, 'A note on locking in a shear flexible triangular plate bending element', Int. j. numer. methods eng., 19, 305-309 (1983).

9. T. Belytschko, H. Stolarski and N. Carpenter, 'A $\mathrm{C}^{\circ}$ triangular plate element with onepoint quadrature', Int. j. numer. methods eng. (to appear).

10. G. Prathap, 'Field consistency and the finite element analysis of multi-field structural problems' in Analysis of Structures (eds. K. A. V. Pandalai and B. R. Somashekar) NAL-SPL-RPT-1/84, Bangalore, 1984.

11. G. Prathap and G. R. Bhashyam, 'Reduced integration and the shear flexible beam element', Int. j. numer. methods eng., 18, 195-210 (1980).

12. G. Prathap and S. Viswanath, 'An optimally integrated 4-node quadrilateral plate bending element', Int. $j$, numer. methods eng., 19, 831-840 (1983).

13. G. Prathap, 'The poor bending response of the four-node plane stress quadrilateral', Int. j. numer. methods eng., 21, 825835 (1985).

14. G. Prathap, 'The curved beam/deep arch/finite ring element revisited', Int. j. numer. methods eng., 21, 389-407 (1985).

15. G. Prathap, 'An additional stiffness parameter measure of error of the second kind in the finite element method', Int. $j$. numer. methods eng., 21, 1001-1012 (1985).

16. T. J. R. Hughes, R. L. Taylor and W. Kanoknukulchal, 'A simple and efficient finite element for plate bending', Int. $j$. numer. methods eng., 11, 1529-1543 (1977).

17. S. Timoshenko and S. Woinowsky-Krieger, Theory of Plates and Shells, 2nd edn, McGraw-Hill, New York, 1959, pp. 501-506.

18. T. Y. Yang, 'High order rectangular shallow shell finite element', J. Eng. Mech. Div., ASCE, 99(EM1), 157-181 (1973). 